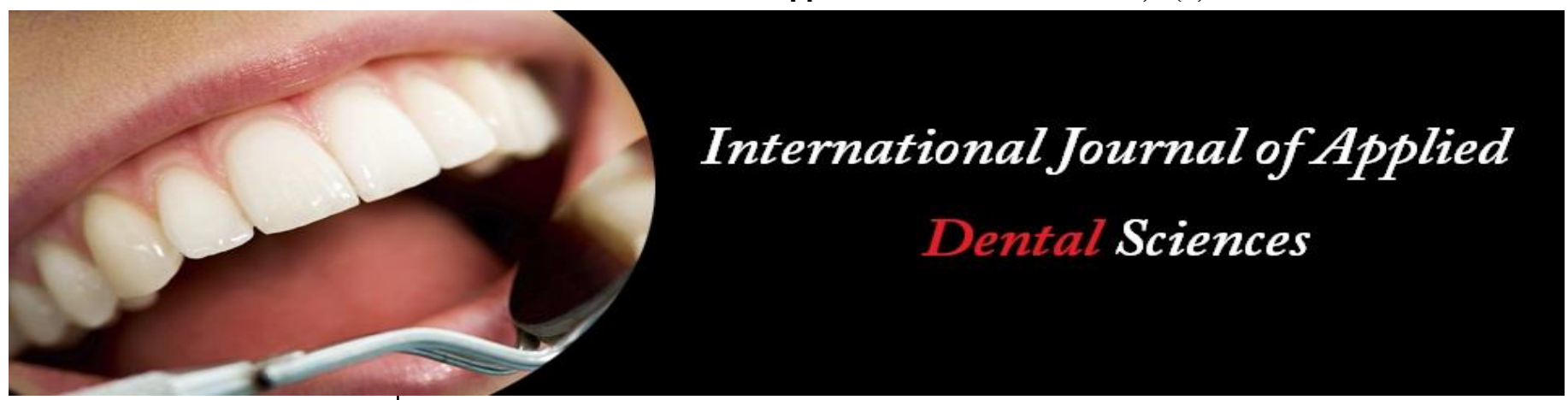

ISSN Print: 2394-7489

ISSN Online: 2394-7497

IJADS 2021; 7(2): 220-224

(C) 2021 IJADS

www.oraljournal.com

Received: 16-01-2021

Accepted: 05-03-2021

\section{Dr. Rajat Nahar}

Post Graduate student,

Department of Prosthodontics

Crown and Bridge, Government

Dental College and Hospital

Mumbai, Maharashtra, India

Dr. Jyoti Tembhurne

Professor and Head,

Department of Prosthodontics

Crown and Bridge, Government

Dental College and Hospital

Mumbai, Maharashtra, India

Dr. Arti Parag Gangurde

Associate Professor, Department

of Prosthodontics Crown and

Bridge, Government Dental

College and Hospital Mumbai,

Maharashtra, India

Dr. Manish R Chauhan

Associate Professor, Department of Prosthodontics Crown and

Bridge, Government Dental

College and Hospital Mumbai,

Maharashtra, India

Dr. Nikhil Kule

Post Graduate Student,

Department of Prosthodontics

Crown and Bridge, Government

Dental College and Hospital

Mumbai, Maharashtra, India

Dr. Vipul Kumar Govindrao

Diwan

Post Graduate Student,

Department of Prosthodontics

Crown and Bridge, Government

Dental College and Hospital

Mumbai, Maharashtra, India

Corresponding Author:

Dr. Rajat Nahar

Post Graduate student,

Department of Prosthodontics

Crown and Bridge, Government

Dental College and Hospital

Mumbai, Maharashtra, India

\section{Full mouth rehabilitation of a patient by replacing maxillary arch with fixed prosthesis opposing cast partial denture in mandibular arch: A case report}

\author{
Dr. Rajat Nahar, Dr. Jyoti Tembhurne, Dr. Arti Parag Gangurde, Dr. \\ Manish R Chauhan, Dr. Nikhil Kule and Dr. Vipul Kumar Govindrao \\ Diwan
}

DOI: https://doi.org/10.22271/oral.2021.v7.i2d.1211

\section{Abstract}

The objective of full mouth rehabilitation is to restore teeth in physiological and functional harmony. Comprehensive treatment planning of multiple missing teeth is challenging, especially in cases of reduced vertical dimension (VD) of occlusion. This case report describes the sequential methodical interdisciplinary approach employed to treat multiple missing teeth with a loss of VD. An interim prosthesis was delivered to newly established restorative vertical dimension. After the validation of new VD, reconstruction was performed with fixed prosthesis in maxillary arch and cast partial denture in mandibular arch. With proper diagnosis and treatment planning dentist can provide functional harmony and esthetic comfort to the patient, thereby improving patient's quality of life.

Keywords: Full mouth rehabilitation, reduced vertical dimension, cast partial denture, provisional restorations

\section{Introduction}

Full mouth rehabilitation employs all diagnostic, therapeutic and restorative procedures for the replacement of lost oral structures. It involves a number of prosthodontic considerations such as analysis of the existing occlusion and occlusal plane; availability of freeway space; size and location of edentulous areas; number, position and condition of the teeth in each arch; the need for altering the vertical dimensions and restoration of function and esthetics ${ }^{[1]}$.

Full Mouth rehabilitation seeks to convert all unfavourable forces on the teeth which inevitably induce pathological conditions, into favourable forces which permit normal function and therefore induce healthy conditions ${ }^{[2]}$.

This clinical report describes the esthetic and functional rehabilitation of a patient with vertical loss due multiple missing teeth. Complete oral rehabilitation was successfully done by integrating the concepts of fixed prosthodontics and removable prosthodontics.

\section{Case report}

A 52 year old female patient reported to department of prosthodontics with chief complaint difficulty in chewing food due to multiple missing teeth. Patient had hypothyroidism and was under medication for the same since two years.

On extra oral examination, TMJ showed no deviation from normal movement and no clicking sound. Lymph nodes appeared normal on palpation. Patient had convex facial profile with decreased lower facial height. She had angular cheilitis around the corners of lips due to loss of vertical dimension.

On intra oral examination, it was observed that patient had fair oral hygiene. Teeth present were $11,12,14,16,17,21,23,26,33,34$ and 35 . There were root pieces with respect to 22 and 32. Post root canal treated crowns with 11, 21 and 16. Mesial caries with respect to 12. Mandibular ridge was knife edged. (Figure 1) Teeth loss were gradually due to caries and mobility (periodontal bone loss) over a period of 8-10 years. 
The remaining teeth had physiologic mobility with good periodontal support. She has been undergoing oral prophylaxis once in two year. This was a diagnosed case of partially edentulous maxillary and mandibular arch with loss of vertical dimension with multiple missing teeth and knife edge ridge over edentulous mandibular arch.

Patient was informed about the need for few extraction, crown lengthening and replacement of the teeth. Various treatment options like removable partial denture, cast partial denture, implant supported removable prosthesis and implant supported fixed prosthesis were explained to the patient and the advantages and disadvantages were discussed. Patient was not willing for implant supported fixed or removable prosthesis and opted for a fixed prosthesis in maxillary arch opposing cast partial denture in mandibular arch.

For the maxillary arch, extraction of 22, surgical crown lengthening with 26, followed by esthetic correction of remaining teeth with crowns and fixed dental prosthesis. In the mandibular arch, fabrication of cast partial denture was planned.

A preliminary impression was made and casts were fabricated. Maxillary cast was mounted on semi adjustable arcon articulator (Hanau wide vue) via face-bow transfer. (Figure 2) A tentative jaw relation (figure 2) was made with new established vertical dimension in centric relation and mandibular cast is mounted on articulator. (Figure 3).

Teeth setting (figure 3) and try-in was done on planned restorative vertical dimension and was evaluated intraorally for esthetics, phonetics, function and comfort. An interim partial denture was fabricated and prosthesis was delivered to the patient for a month. (Figure 4) A month later, patient comfort and TMJ joint was evaluated for tenderness or clicking. Patient was comfortable with the new restorative vertical dimension. Crown removal was done with respect to 11, 21 and 16. A second hydrocolloid impression was made for upper arch and lower arch with partial denture in mouth. An orientation relation with a face-bow record was taken to mount maxillary cast and interocclusal record to mount mandibular cast with previously established restorative vertical dimension in centric relation. A protrusive record was taken to adjust condylar guidance. The condylar guidance on both sides was $25^{\circ}$ and lateral inclination value was obtained $15^{\circ}$ using formula $\mathrm{L}=\mathrm{H} / 8+12$. A wax mock-up was done for maxillary arch in established vertical and centric. (Figure 5) The maxillary teeth were prepared and temporaries were transferred using bis-acrylate resin (Luxatemp). Temporaries were adjusted intraorally and checked for esthetics, function and comfort of the patient. (Figure 6) These temporaries remained in function for a month to assess patient's comfort. A third hydrocolloid impression was made for maxillary arch with cemented provisional restorations and jaw relation on wax rim on mandible with same established vertical relation. Maxillary cast was mounted using face-bow transfer. The mandibular teeth $33,34,35$ were then prepared to receive a full veneer crown. Impression was made with addition silicone using putty wash technique (Honigum putty and light body) and chemo-mechanical retraction of gingiva of the prepared teeth. A jaw relation record was made using wax rims for edentulous areas and Aluwax for dentulous areas. (Figure 7) Mandibular cast was mounted on semi-adjustable articulator. Wax pattern was fabricated with cingulum rest on 33 and distal and mesial rest on 34 and 35 respectively after surveying. Metal try-in done followed by bisque trial and final prosthesis is cemented using glass ionomer luting cement (GC India). After a week, medium body impression was made for mandibular arch using a custom tray over which tray adhesive was applied and multiple vent holes were made for fabrication of cast partial framework. Also, in maxilla temporaries are removed and chemico-mechanical retraction was done and impression was made using addition silicon using putty wash technique. First the mandibular cast was mounted with reference to previously mounted maxillary cast in centric bite, then maxillary cast was mounted in relation to mandibular cast using sectional bite on mandibular wax rim on both left and right side. Cast partial framework was fabricated for mandibular arch with canine rest and embrasure clasp on both premolars. Framework assembly for CPD was checked for fitting intraorally after that cold cure acrylic resin (Maarc) was adapted over edentulous area for making functional impression. Border moulding was done using low fusing compound (DPI) and final impression was made using light body condensation silicon (Zhermack). Casts were poured using altered cast technique (Figure 8) and mounted in semiadjustable arcon articulator. Meanwhile, metal try-in followed by bisque trial was carried for maxillary arch. Teeth setting was done for mandibular arch and trial was done in mouth to evaluate for esthetics, phonetics, function and occlusion. (Figure 9) Acrylisation for CPD is done using heat cure acrylic resin and was finished and polished. Final bridge were cemented in maxillary arch using GIC luting cement, and mandibular cast partial denture was delivered to the patient. (Figure 10) Patient was recalled for follow-up after a week and then one month followed by once in a six month.

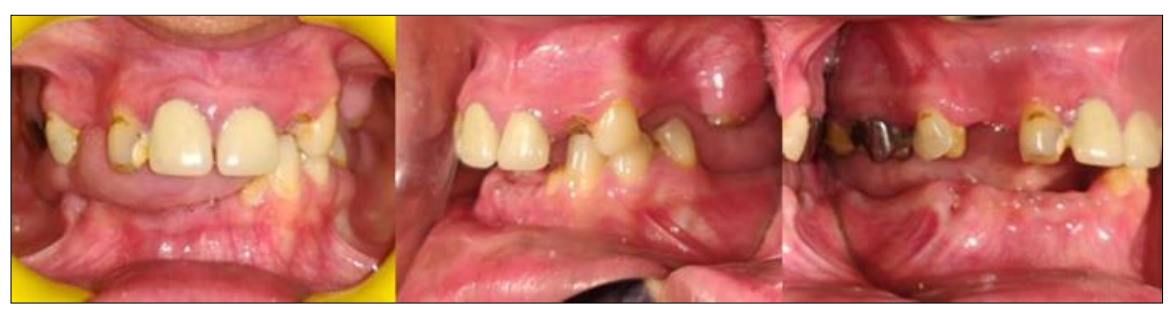

Fig 1: Intraoral pre-operative

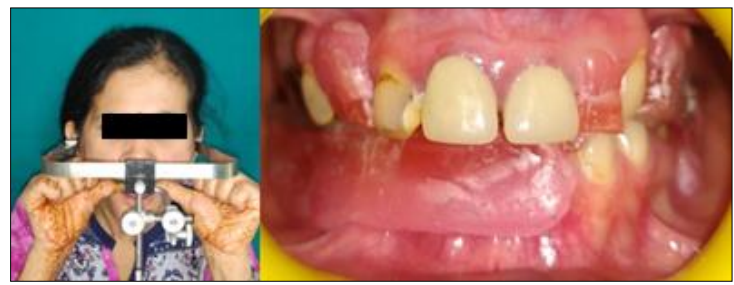

Fig 2: Face bow record and tentative jaw relation 


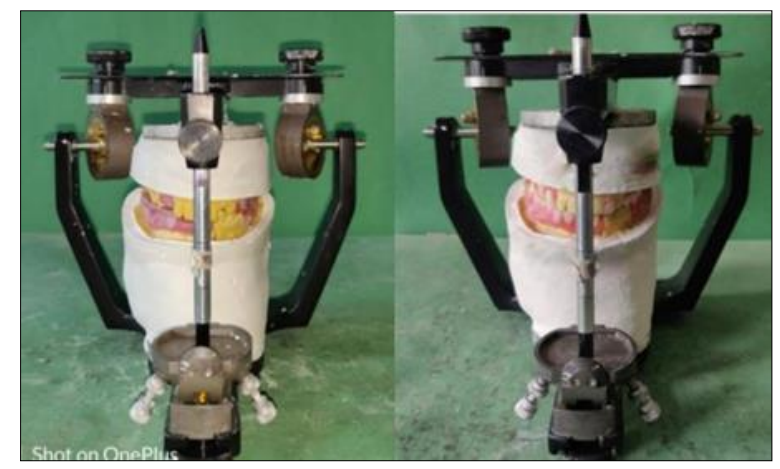

Fig 3: Mounting on semi-adjustable articulator and teeth arrangement

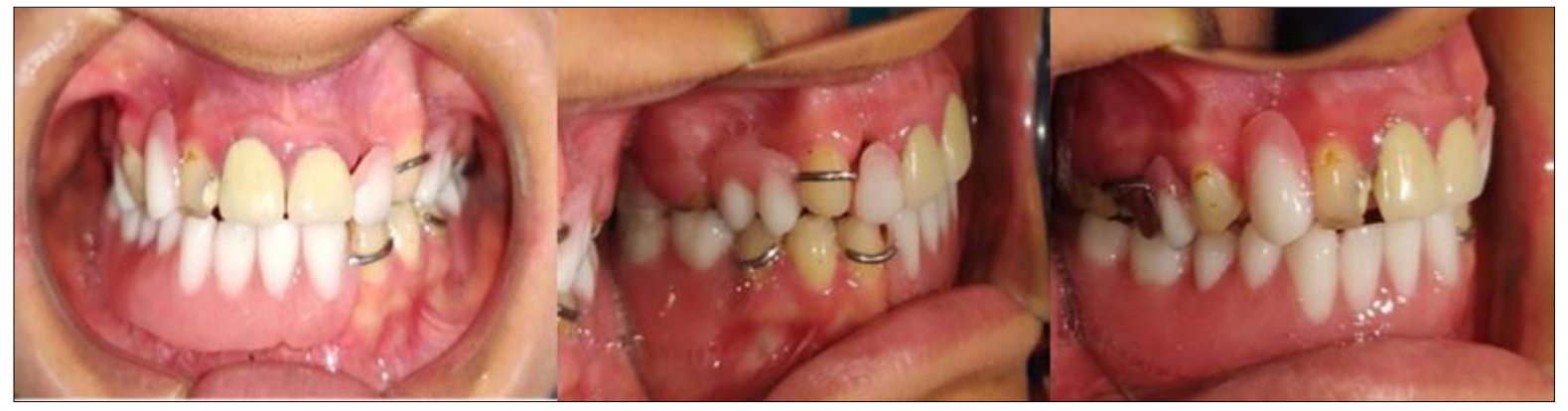

Fig 4: Interim partial denture

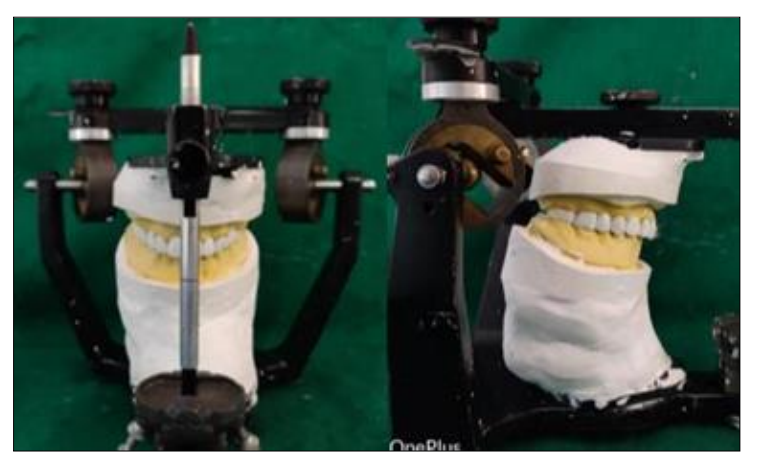

Fig 5: Wax mock-up

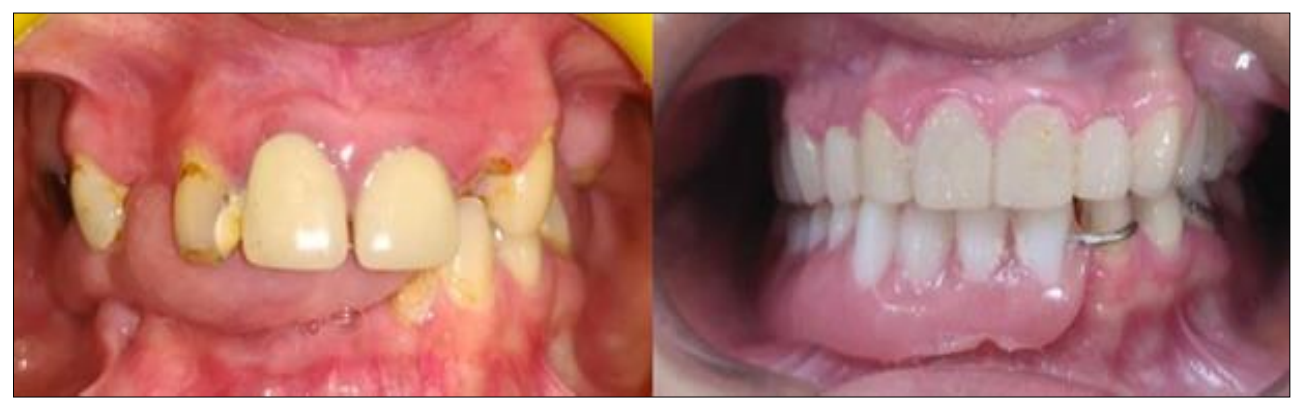

Fig 6: Temporisation

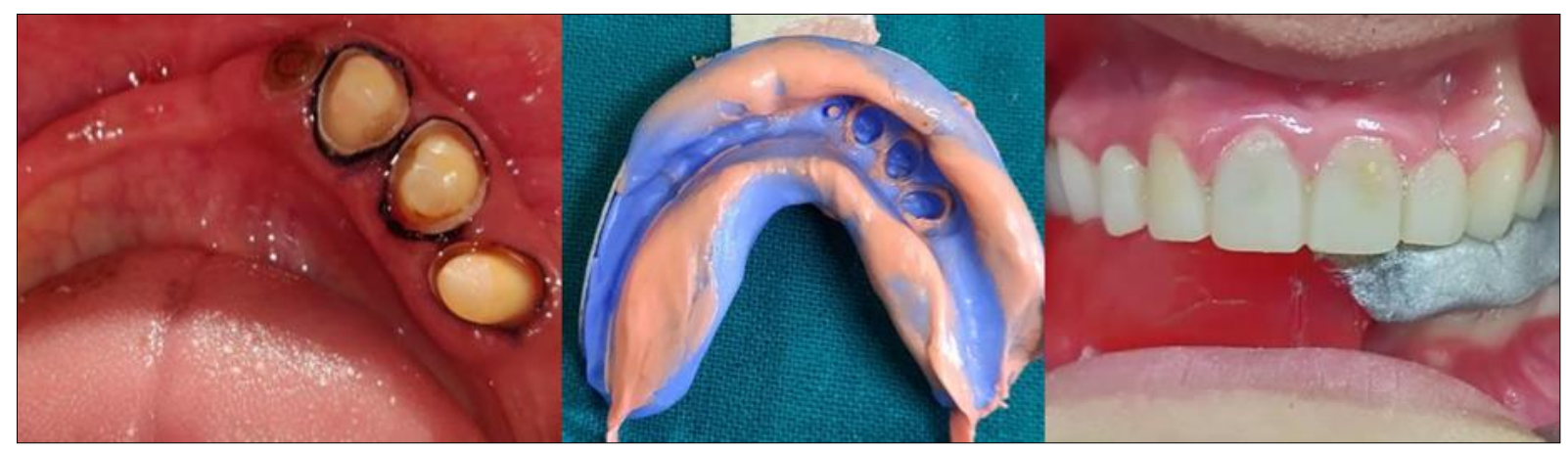

Fig 7: Tooth preparation in mandibular arch, putty wash impression and jaw relation 


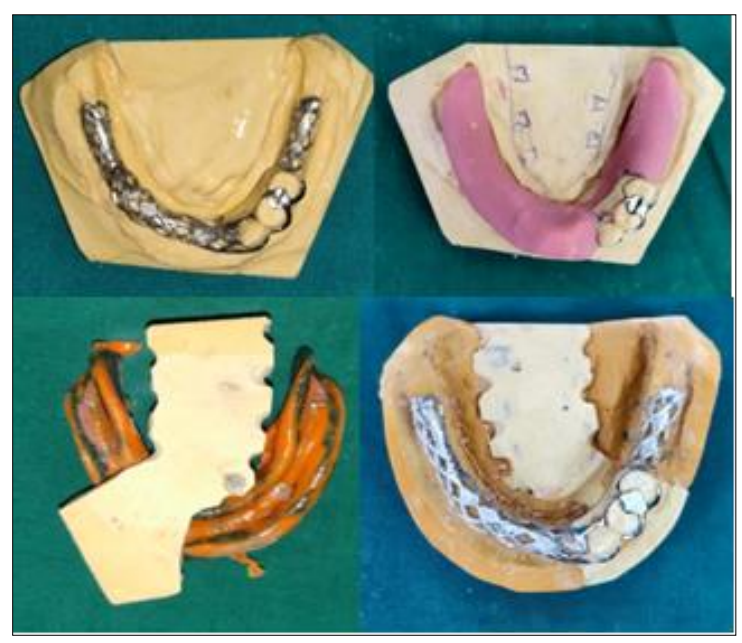

Fig 8: Cast partial frame-work and altered cast technique

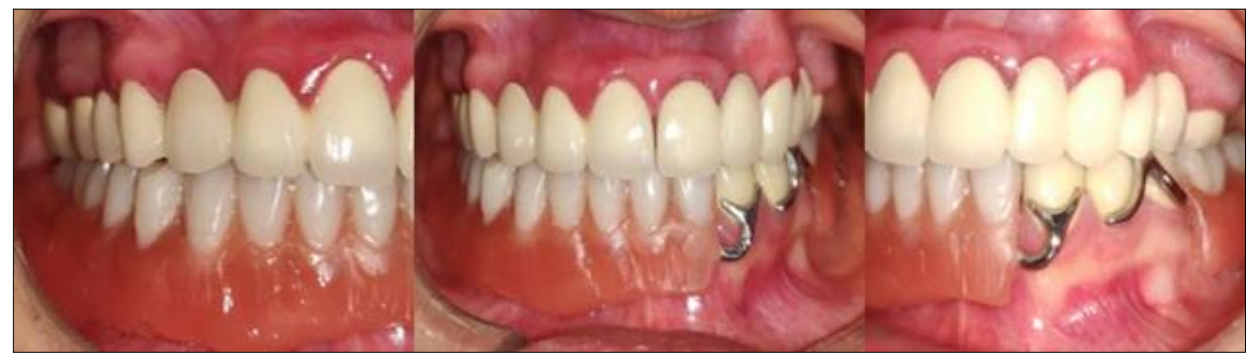

Fig 9: Final try-in for maxillary fixed prosthesis and mandibular cast partial denture
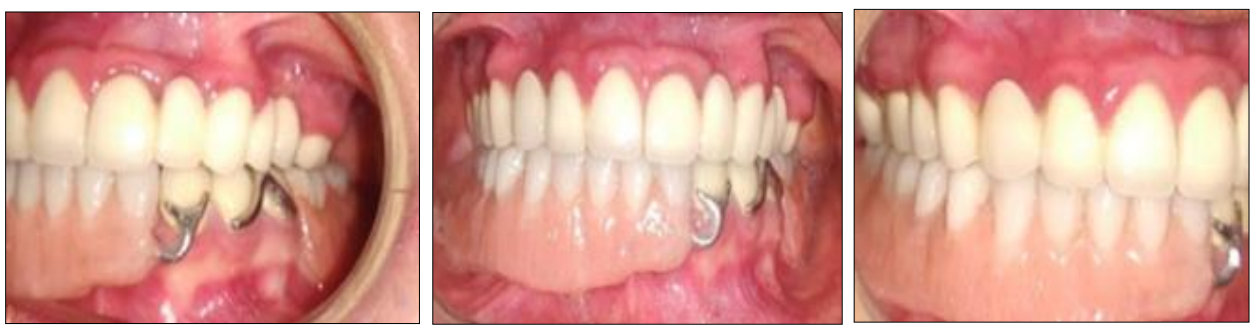

Fig 10: Final prosthesis delivered

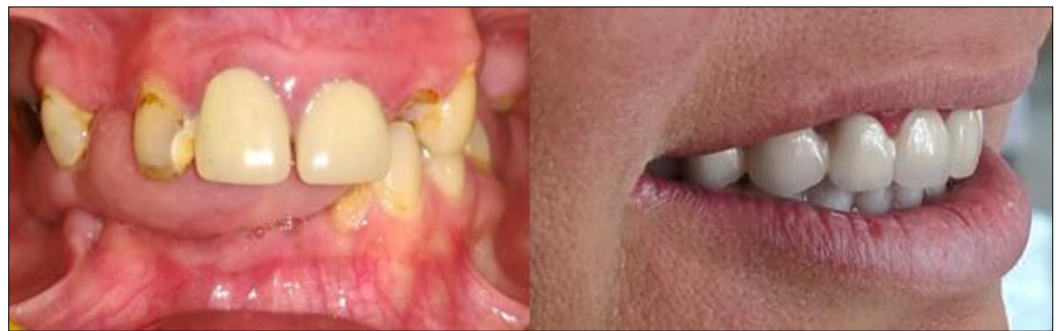

Fig 11: Pre-operative and post-operative

\section{Discussion}

The case report presented in this article describes a patient with multiple missing teeth and decreased VDO. The management of the mandibular arch with distal extension having knife edge ridge is a restorative challenge, and often presents in association with reduced VD, which further complicates the rehabilitation procedures. As the patient cannot afford dental implants, the treatment plan was executed on a simplified and efficient rehabilitation technique so that low-cost rehabilitation can be done with great possibility of longevity.

The reduction of VDO in the present case might be because of the long neglect of the edentulousness. As this case presented with a huge challenge of lack of restorative space, the VDO was increased for the best functional and esthetic anterior contact in centric. According to Carlsson, increase in vertical dimension within physiologic limits will not have hazardous effects on the masticatory apparatus of the patient ${ }^{[3]}$. Also the transitional prosthesis that was fabricated on the proposed vertical dimension allowed validation of VDO and functional occlusion prior to initiation of definitive CPD ${ }^{[4]}$. This trial phase of wearing transitional RPD allows neuromuscular adjustment to a change in VDO and helps to determine patient's comfortability with the increased VDO ${ }^{[5]}$. The minimum recommended period for adaptation to this increased VDO has been determined to be $6-8$ weeks ${ }^{[6]}$.

The longitivity of prosthesis depends on clinician and the patient. In case of fixed prosthesis appropriate good crown preparation, selection of material and impression technique by the clinician play vital role ${ }^{[7]}$. Meticulous follow up regime 
comprising of proper clinical and radiographical examination, personalised preventative protocol prescription for the patient can further add to the life of prosthesis ${ }^{[8]}$.

\section{Conclusion}

Full mouth rehabilitation requires the proper implication of interdisciplinary concepts to achieve functional and esthetic success. Diagnosis and treatment plan should aim at deciding the need of altering the VD for meeting the biologic, restorative, function and esthetic requirements. With proper treatment planning dentists can improve patients quality of life.

\section{References}

1. Dua P, Singh JP, Aghi A. Aesthetic and functional rehabilitation of a case of mutilated dentition and loss of vertical dimensions. The Journal of Indian Prosthodontic Society 2011;11(3):189.

2. Pasam N, Raja S. Full mouth rehabilitation. Indian J Dent Adv 2010;2:285-90.

3. Carlsson GE, Ingervall B, Kocak G. Effect of increasing vertical dimension on the masticatory system in subjects with natural teeth. J Prosthet Dent 1979;41(3):284-9.

4. Jaikumar RA, Madhulika N, Kumar RP, Vijayalakshmi K. Prosthetic rehabilitation in a partially edentulous patient with lost vertical dimension: A case report. J Indian Acad Dent Spec Res 2014;1:70-3.

5. Jahangiri L, Jang S. Onlay partial denture technique for assessment of adequate occlusal vertical dimension: A clinical report. J Prosthet Dent 2002;87:1-4.

6. Patel MB, Bencharit S. A treatment protocol for restoring occlusal vertical dimension using an overlay removable partial denture as an alternative to extensive fixed restorations: A clinical report. Open Dent J 2009;3:213-8.

7. Bell AM, Kurzeja R, Gamberg MG. Ceramometal, crowns, bridges. Focus on failures. Dent Clin North Am 1985;29(4):763-778.

8. Johansson A, Johansson AK, Omar R, Carlsson GE. Rehabilitation of the worn dentition. Journal of oral rehabilitation 2008;35(7):548-66. 